\title{
Los Angeles's Perspective of COVID 19 Effect and Impact on Cardiac Surgery
}

\author{
Tiffany Worthington ${ }^{1}$ and Ali Khoynezhad ${ }^{1}$ \\ ${ }^{1}$ Memorial Care Heart and Vascular Institute
}

August 23, 2020

\begin{abstract}
Coronavirus 2019 (COVID 19) captured worldwide attention as the virus spread from initial detection as a pneumonia of unknown cause in Wuhan, China in December 2019 to the declaration of a pandemic by the World Health Organization only three months later. This paper describes the impact of COVID 19 on cardiac surgery services in the greater Los Angeles area. Discussion includes public health response to the pandemic, how local hospitals reacted to the expected surge in COVID patients, establishment of telemedicine services, and the projected future impact on California healthcare as a result of the COVID 19 pandemic.
\end{abstract}

\section{Los Angeles's Perspective of COVID 19 Effect and Impact on Cardiac Surgery}

Tiffany Worthington, DMSc, PA-C and Ali Khoynezhad, MD, PhD

Department of Cardiovascular Surgery

MemorialCare Heart and Vascular Institute

Running Title: COVID-19 in Los Angeles

Corresponding author:

Ali Khoynezhad, MD, PhD

Department of Cardiovascular Surgery

MemorialCare Heart and Vascular Institute

2801 Atlantic Ave

Long Beach, CA 90806

akhoynezhad@memorialcare.org

Phone: 562-933-7174

Fax: 562-933-7178

Author contributions:

A.K. and T.W. both contributed to data collection, drafting the article, critical revision of the article. A.K. performed final approval of the version to be published.

This manuscript did not involve research of human subjects, therefore is exempt from Institutional Review Board (IRB) or Ethics Committee Approval. 


\section{Los Angeles's Perspective of COVID 19 Effect and Impact on Cardiac Surgery}

Los Angeles County is home to over 10 million residents. As comparison only nine states in the US have more population than Los Angeles county. It serves as home to professional sporting teams, the entertainment industry, theme parks, and a variety of outdoor recreation activities. LA County is a popular destination for approximately 48.3 million tourists annually.

On December 31, 2019, an initial report of pneumonia of unknown cause was reported to the World Health Organization (WHO) office detected in Wuhan in the Hubei province of China. By January 30, WHO declared a "public health emergency of international concern" as COVID-19 cases had been reported in five WHO regions in the course of one month.[1] That same day, LA Times headlines read "For Americans, flu remains a bigger threat than coronavirus".[2] As a community, LA was still recovering from the news of Kobe Bryant and his daughter dying in a helicopter crash along with several other passengers, headlines of novel coronavirus spread were buried within the newspapers, with limited appearance on the front page.

As concerns worldwide continued to grow surrounding the COVID-19 pandemic, WHO issued a notice of impending shortage of personal protective equipment (PPE) on March 3, 2020. [3] The following day, LA county officials issued a declaration of local public health emergency. By 5 days later, the first community spread case was reported in LA. On March $11^{\text {th }}$, the first death from COVID-19 was reported in Los Angeles County. [4] March $18^{\text {th }}$ Governor Newsom wrote to President Trump requesting federal funding and additional support with concern that more than half of the population of California would be infected with the virus over the following 2-month period. As awareness of the impact of the pandemic in the greater LA area continued, a "safer at home" emergency order was issued on March $19^{\text {th }}$. This order entailed the closure of all non-essential businesses, no gathering of more than 10 people, and all persons to remain home unless visiting an essential business or participating in outdoor activities at least 6 feet apart from others. The order to wear face masks whenever out of the home and interacting with people was issued as an official guidance on April 1 and became a requirement on April 10 ${ }^{\text {th }}$. On April 23 ${ }^{\text {rd }}$, COVID became the leading cause of death in LA county, surpassing coronary artery disease, emphysema, influenza, and COPD. [4]

As the number of COVID cases continued to grow, certain trends were emerging, including a significant impact on residential care facilities and in lower-income areas. In response to a growing shortage of staffing in nursing homes, the medical director for LA county EMS made a temporary allowance for EMTs and paramedics to staff the nursing homes. As of mid-June, there were 321 nursing homes in LA county with a least one resident who has tested positive for COVID. [5] The number of deaths related to COVID have been three times higher in areas of poverty than in higher income areas within LA county. Ninety-four percent of deaths in the LA area related to COVID were patients with underlying health conditions. Of those who died, $42 \%$ occurred among Latino residents, $28 \%$ White, $17 \%$ Asian, $11 \%$ African American, and less than $1 \%$ Native Hawaiian/Pacific Islander residents. [6]

\section{Surge Planning}

Convention centers and arenas in the area were converted to field hospitals to handle the predicted patient surge. The US Naval Ship Mercy, a Navy hospital ship with patient capacity of 1,000 beds was dispatched to the Port of Los Angeles where it remained for seven week before returning to its homeport in San Diego. The ship treated 77 patients with non-COVID medical complaints during the deployment. California Health Corps, a state-wide volunteer program was initiated which yielded substantial interest, 82,000 volunteers signed up in the first week.

Surge planning was initiated with emergency triage drills, development of labor pools, immediate cessation of scheduling elective cases, and daily policy updates were provided via email communications across the healthcare system. In preparation for numerous patients coming to the emergency department, designation of zones within departments for persons with suspected COVID, confirmed COVID, and those who were being treated for non-related medical conditions. Online dashboards were created for reporting daily hospital capacities throughout the county for up-to-date status monitoring. 
Emergency medical services developed interventions to minimize unnecessary patient volume in emergency departments by aggressive field triage measures including postponing transport of cardiac arrest patients for at least five minutes after revival on scene and establishing a videoconferencing platform for emergency department physicians to provide telemedicine services to patients at home and managing non-urgent issues remotely.

\section{Cardiac Surgery}

The transition from standard operative practice to complete shutdown of elective cases was immediate in mid-March. Operating room boards were wiped clean as hospital administration scrambled to devise a protocol for triaging which cases would be appropriate to continue as planned and which cases could be safely postponed, minimizing PPE use and maximize available resources. Daily updates were issued reporting number of COVID cases within the healthcare organization, county, and state. Initial projections for the LA county area impact provided an expected two week timeline to exceeding ICU bed and ventilator capacity. During this time, surgical cases were restricted to emergencies only. Aortic dissections and patients with severely symptomatic valve disease were among the only cardiac surgical cases to be performed. Over the following month, the number of COVID patients fell drastically short of predicted. Throughout the state, there was a $62 \%$ decline in ED visits in April and a $35 \%$ decline in total hospitalizations when compared to previous years. [7-8] Triaging protocols were developed to begin a controlled re-integration of services in the cardiac surgery unit while actively monitoring COVID patient volume, ICU bed capacity, and availability of PPE. In mid-April, surgery services were resumed with team-based decision making on which cases could safely continue to be postponed versus those that took priority to be managed as early as possible. Patients with symptomatic disease have been amenable to proceeding with surgery, however some with asymptomatic disease such as aortic aneurysm have shown hesitation in rescheduling their previously planned surgery. Some changes to the pre-operative process include COVID testing within 48 hours of surgery and the restriction of visitors within the hospital. IPads are used to facilitate patient communication with their family members during their hospital stay, and daily telephone updates to family members, and Facetime communication between the patients and respective family members became part of the new routine.

\section{Outpatient Response}

As news spread of the COVID pandemic, patients became hesitant to come to the outpatient clinic. During the first few weeks, patients were hesitant to participate in videoconferencing visits, however by April, telemedicine visits became routine practice. Nationally, telemedicine prior to COVID accounted for $0.25 \%$ of medical services provided to Medicare beneficiaries. [9] With the emergency passage of waiver 1135 and Coronavirus Preparedness and Response Supplemental Appropriations Act, prohibitive barriers in telemedicine were eliminated allowing for rapid expansion to continue managing patients that are safer at home during a pandemic. During peak telemedicine service between March and April, $45 \%$ of all patient encounters were performed remotely using a videoconferencing platform within our healthcare organization. With the simultaneous interruption of cardiology and diagnostic imaging services, new referrals during the restriction period were limited.

An active surveillance program was initiated for all patients who had been scheduled for surgery that had been cancelled due to hospital restrictions. Patient check-ins were performed on a weekly basis with videoconferencing and telephone visits to evaluate for changes in symptoms or progression of disease necessitating more immediate intervention.

In mid-May, as outpatient services continued ramping up, the two-month backlog of patients who had declined telemedicine visits started to return for follow up. Currently, chronic aortic dissection and aortic aneurysm patients have been the most challenging to recapture, with $30 \%$ requesting deferral of their routine imaging to a later date.

\section{Future Impact}

The re-introduction of social activities in the LA area has been cautious and closely monitored. Initially 
hiking trails and beaches were opened for recreational use mid-May. Near the end of May, restaurants were permitted to reopen with the agreement that certain safety protocols were met such as outside seating, maintantince of 6 -feet distance, offering electornic menu, and hand hygiene protocols for employees. Health officials inspected over 2,000 LA restaurants two weeks after opening and found that approximately $50 \%$ were not in compliance with the safety protocols issued.

As of June 20, 890,793 have been tested out of a total population of over 10 million residents. [5] There have been 79,609 confirmed cases with 3,063 deaths. [5] Of these 7,632 have required hospitalization. [5] The predicted surge has not happened to date, and this may have to do with early state and county-wide shutdown, however a progressive and gradual increase of COVID-19 postive cases has been occurring recently. As the LA area continues to relax restrictions, health officials closely monitor for changes indicating the need to resume at-home restrictions.

The next crisis facing California hospitals will likely be the financial impact of cancelling elective cases, costs related to surge preparation, a ravaged economy, and a predicted increase in uninsured or underinsured patients as job losses push people towards Medi-Cal, the California Medicaid insurance coverage. The University of California health system reported losses over $\$ 140$ Million over 6 academic health centers from the cancellation of elective procedures. Across California, hospitals are reporting average losses of 20-30\% with a predicted net loss of up to $\$ 14.6$ billion across about 400 hospitals by the end of 2020. [8] In addition to direct losses from COVID impact, California passed a state law requiring hospitals to update earthquake preparedness infrastructure by 2030, which was estimated to cost up to $\$ 100$ billion over the next 9 years. $[10]$

\section{References}

1. World Health Organization. Statement on the Second Meeting of the International Health Regulations. Emergency Committee regarding the outbreak of novel coronavirus (2019-nCoV); 2005. InURL: https://www. who. int/news-room/detail/30-01-2020-statement-onthe-second-meeting-of-theinternational-health-regulations.

2. Karlamangla S. For Americans, flu remains a bigger concern than coronavirus. LA Times. 31 Jan. 2020

3. World Health Organization. Shortage of personal protective equipment endangering health workers worldwide. 3 March 2020. https://www.who.int/news-room/detail/03-03-2020-shortage-of-personalprotective-equipment-endangering-health-workers-worldwide

4. County of Los Angeles Public Health. Los Angeles County announces 68 new deaths related to 2019 novel coronavirus (COVID-19); 1081 new cases of confirmed COVID-19 in Los Angeles county. [press release] 23 April 2020

5. County of Los Angeles Public Health. LA county COVID-19 by the numbers. 23 June 2020

6. County of Los Angeles Public Health. Los Angeles County announces 34 new deaths related to 2019 novel coronavirus (COVID-19) - 2,364 new cases of confirmed COVID-19 in Los Angeles county. [press release] 23 June 2020

7. Office of Statewide Health Planning and Development. Hospital annual financial disclosure report FY17-18.

8. KaufmanHall. The financial impact of COVID-19 on California hospitals - CHA member survey. June 2020

9. Centers for Medicare \& Medicaid Services. Information on Medicare telehealth. CMS. 18 November 2018

10. Preston, Benjamin Lee, Tom LaTourrette, James R. Broyles, R. J. Briggs, David Catt, Christopher Nelson, Jeanne S. Ringel, and Daniel A. Waxman, Updating the Costs of Compliance for California's Hospital Seismic Safety Standards. Santa Monica, CA: RAND Corporation, 2019. 